\title{
Mild and moderate cognitive impairment and mortality among Brazilian older adults in long-term follow-up: The Bambui Health Aging Study
}

\author{
Cecilia G. Campos, ${ }^{1}$ iD Breno S. Diniz, ${ }^{2,3}$ iD Josélia O. Firmo, ${ }^{1}$ (D) M. Fernanda Lima-Costa, ${ }^{1,4}$ (iD \\ Sergio L. Blay, ${ }^{5}$ iD Erico Castro-Costa ${ }^{1}$ iD \\ ${ }^{1}$ Núcleo de Estudos em Saúde Pública e Envelhecimento, Instituto René Rachou, Fundação Oswaldo Cruz, Belo Horizonte, MG, Brazil. \\ ${ }^{2}$ Department of Psychiatry, Faculty of Medicine, University of Toronto, Toronto, ON, Canada. ${ }^{3}$ Geriatric Psychiatry Division, Center for \\ Addiction and Mental Health, Toronto, ON, Canada. ${ }^{4}$ Departamento de Medicina Preventiva e Social, Faculdade de Medicina, Universidade \\ Federal de Minas Gerais, Belo Horizonte, MG, Brazil. ${ }^{5}$ Departamento de Psiquiatria, Universidade Federal de São Paulo, São Paulo, SP, \\ Brazil.
}

\begin{abstract}
Objective: To compare mortality in older adults with and without mild or moderate cognitive impairment over 15 years of follow-up in a middle-income country, where little information on this subject is available.

Methods: A total of 1,281 community-dwelling older adults were followed-up for a median of 13.3 years. We evaluated their cognitive impairment using the Mini-Mental State Examination, categorizing it as none (1.0 SD above cutoff means), mild (1.0 SD below cutoff means) or moderate (2.0 SD below cutoff means). The date of death was determined by reviewing death certificates. Cox's proportional hazards models were used to evaluate the risk of mortality in participants with cognitive impairment. Results: Participants with mild or moderate cognitive impairment had a higher mortality risk than those without it in the unadjusted model, but these associations did not remain in the final model. After sex stratification, only men with moderate cognitive impairment had a higher mortality risk in the final model.

Conclusion: The findings suggest an association between moderate cognitive impairment and all-cause mortality in men in a large Brazilian cohort of older adults.
\end{abstract}

Keywords: Older adults; mortality; cognitive impairment; longitudinal study; follow-up

\section{Introduction}

Cognitive impairment is an intermediate stage between normal cognitive aging and dementia. Although diagnosing subclinical cognitive impairment is essential for identifying individuals at high risk of dementia, this task is complex and challenging due to a lack of standardized diagnostic criteria. ${ }^{1}$ Cognitive impairment varies from strict clinical criteria for mild cognitive impairment $(\mathrm{MCl})^{2-5}$ to definitions of cognitive decline used in population-based studies, which cover a broader range of dysfunctions. ${ }^{6-8}$

Six previous longitudinal studies from high-income countries have shown that individuals with cognitive impairment have a higher mortality risk than healthy controls. ${ }^{7-12}$ However, these studies measured cognitive impairment differently. In four of these studies, cognitive impairment was identified by one-time administration of a cognitive functioning scale, ${ }^{6-8,11}$ while in the other two it was diagnosed according to $\mathrm{MCl}$ criteria. ${ }^{9,10,12}$ Data on

Correspondence: Erico Castro-Costa, Núcleo de Estudos em Saúde Pública e Envelhecimento, Instituto René Rachou, Fundação Oswaldo Cruz, Av. Augusto de Lima, 1715, CEP 30190-002, Belo Horizonte, MG, Brazil.

E-mail: ericocastrocosta@gmail.com

Submitted Aug 13 2019, accepted Feb 06 2020, Epub Jun 012020. the association between cognitive impairment and mortality is scarce in low- and-middle-income countries: a single Tanzanian study ${ }^{13}$ applied $\mathrm{MCl}$ criteria and found that, after adjusting for the effects of age, sex, and education, a higher mortality risk was associated with cognitive impairment.

The cognitive decline model used in population-based studies is different from and more prevalent than strict $\mathrm{MCl}$ criteria, including a higher rate of progression to dementia. ${ }^{14,15}$ Consistently lower rates of progression from $\mathrm{MCl}$ to dementia are found in community services than in specialty and clinical research services. ${ }^{16,17}$ Furthermore, the findings of population-based studies indicate that many individuals with $\mathrm{MCl}$ remain stable or even revert to normal during follow-up. ${ }^{16-18}$ Thus, the objective of the current study was to compare mortality in individuals with and without cognitive impairment according to populationbased criteria over 15 years of follow-up in Brazil, a middle-income country.

How to cite this article: Campos CG, Diniz BS, Firmo JO, LimaCosta MF, Blay SL, Castro-Costa E. Mild and moderate cognitive impairment and mortality among Brazilian older adults in long-term follow-up: The Bambui Health Aging Study. Braz J Psychiatry. 2020;42:583-590. http://dx.doi.org/10.1590/1516-4446-2019-0654 


\section{Methods}

\section{Study area and population}

This analysis was carried out using data from a 1997 population-based prospective cohort study on aging in Bambui, a city of approximately 15,000 inhabitants in Minas Gerais, Brazil. The city's United Nations' Human Development Index was 0.70, implying medium development, and its life expectancy was 70.2 years in $1991 .^{19}$ The city has one general hospital and approximately one physician per 1,000 inhabitants. Its main economic activities are agriculture (particularly farming) and commerce. ${ }^{19}$

The procedures of the Bambui Cohort Study of Aging have been described elsewhere. ${ }^{19}$ Briefly, the baseline cohort population consisted of all residents aged 60 years and over on January 1, 1997, who were identified through a private census of the city. Of a total of 1,742 older residents identified in this census, 92.2\% (1,606) were interviewed and $85.9 \%(1,496)$ underwent clinical examination during the baseline survey, which consisted of hematological assays, biochemical assays, and serological tests for Chagas disease, electrocardiogram, and anthropometric and blood pressure measurement.

The interviews lasted approximately $90 \mathrm{~min}$ and were carried out in the participants' homes. Each interview was completed in one or two visits (within 1 week), depending upon the respondent's need for rest. Neither the interviewers nor the respondents were aware of the clinical results at the time of the interview. A sample of $10 \%$ of participants was selected for reliability assessment. Cohort members underwent annual follow-ups, consisting of standardized interviews and death certificate verification.

\section{Mortality data source}

Deaths occurring up to December 31, 2011 were included in this analysis. Deaths were reported by next of kin during the annual follow-up interview and were verified through the Brazilian Ministry of Health's mortality information system. Similar procedures were used to verify the death of individuals who moved away from the study area (5.2\%). The endpoint in this analysis was death from any cause.

\section{Cognitive assessment}

The Bambui Cohort Study of Aging questionnaire included a standard Brazilian version of the Mini-Mental State Examination (MMSE). ${ }^{20}$ In the Brazilian validation study, some questions were modified according to their relevance to the target population. Questions about the season, the building, and the floor in the original orientation section ${ }^{21}$ were replaced with the period of the day, the room, and the address in the Brazilian version. ${ }^{20}$ In the registration and recall section, the words used were gato (cat), árvore (tree), and violão (guitar). In the attention and calculation section, serial fives replaced serial sevens, and for the reverse spelling task, "World" was replaced by "Maria," due to is common usage in Brazilian culture. Previous analysis showed that the MMSE version used in this study had cross-cultural equivalence and invariance or stability over time in its factor structure. ${ }^{22,23}$ According to total MMSE scores, cognitive impairment was classified as none (1.0 standard deviation [SD] above cutoff means), mild (1.0 SD below cutoff means) or moderate (2.0 SD below cutoff means). These groupings were applied to allow comparison with previous research. ${ }^{8}$

\section{Sociodemographic characteristics}

Data were collected on the following variables: age, sex, number of complete years of education ( $<4$ years vs. $\geqslant$ 4 years: 4 years was the statutory primary education for this population), marital status (married or living together vs. single, separated, divorced, or widowed), and selfreported ethnicity (Caucasian vs. non-Caucasian, e.g. African or mixed ethnicity).

\section{Lifestyle variables}

Alcohol consumption was determined through the question: "During the previous 12 months, on average, how often have you drunk alcohol?"; caffeine consumption was determined through the question: "How many cups of coffee, tea, or other caffeinated drinks have you had daily in the last 12 months?" Current smokers were defined as participants who had smoked at least 100 cigarettes in their lifetime and were still smokers. Physical activity level was determined through the question: "During your leisure time in the previous 90 days, how many times did you go for a walk, work out, or participate in sports for at least 20-30 min?"

\section{Functional status and health conditions}

Functional impairment was defined as the inability to independently perform at least one of the following five activities of daily living: dressing, walking across a room, bathing or showering, eating, getting in or out of bed, and using the toilet. Body mass index (BMI) was assessed in a visit to the Bambui field clinic using the standard protocol. Diabetes mellitus was defined as a fasting blood glucose level $\geqslant 126 \mathrm{mg} / \mathrm{dL}$ and/or current use of insulin or oral antidiabetic drug treatment. ${ }^{24}$ Infection with $T$. cruzi was assessed by two parallel methods. Infection was defined as seropositivity in both indirect hemagglutination assay (Biolab-Mérieux, Rio de Janeiro, Brazil) and enzymelinked immunosorbent assay (Abbott, São Paulo, Brazil). Information on lifetime history of stroke was obtained by interview according to methods described elsewhere. ${ }^{25}$ Hypertension was defined as systolic blood pressure $\geqslant 140 \mathrm{mmHg}$ and/or diastolic blood pressure $\geqslant 90 \mathrm{mmHg}$ and/or the use of antihypertensive drugs. ${ }^{26}$ Genomic DNA for APOE genotyping was extracted from blood samples using a Wizard ${ }^{\circledR}$ Genomic DNA Purification System (Promega, Madison, USA). In the present analysis, we considered three groups of gene composition for the $\varepsilon 4$ allele: absent, homozygous $(\varepsilon 4 \varepsilon 4)$, and heterozygous (42.8\% is $\varepsilon 2 \varepsilon 4$ and $57.2 \%$ is $\varepsilon 3 \varepsilon 4)$.

Depressive symptoms were assessed by the General Health Questionnaire-12, using a cutoff of $4 / 5$ to define case-level symptomatology. ${ }^{27}$ 


\section{Statistical analysis}

The follow-up period was from the date of the first examination until the date of death or the study endpoint (December 31, 2011). Mortality rates were estimated using the person-time method the (number of deaths divided by person-years of follow-up). Hazard ratios (HR) for death in each cognitive impairment category were estimated by Cox's proportional hazards regression modeling. Gender, years of education, being unmarried (including separated, divorced, or widowed status), selfreported ethnicity, alcohol consumption, smoking, exercise, physical function, diabetes mellitus, infection with T. cruzi, stroke, hypertension, APOE \&4 allele, and depressive symptoms were used as categorical measures, while age, caffeine consumption, and BMI were used as continuous measures.

The analysis was based on four models, which were performed for the total sample and then stratified according to gender. First, we estimated the crude association between cognitive impairment and mortality, and then adjusted incrementally for 1) demographic variables, 2) lifestyle, and 3) health measures. A formal test of the proportional-hazards assumption based on Schoenfeld residuals was also performed. All analyses were performed in Stata version 14.1.

\section{Ethics}

The project was approved by the ethics committee of the Fundação Oswaldo Cruz, Brazil.

\section{Results}

The mean follow-up duration was 11.0 years (median: 13.3), corresponding to $14,120.4$ person-years at risk. A total of 639 subjects died during follow-up (crude mortality rates for mild and moderate cognitive impairment were 69.1 per 1,000 person-years and 93.9 per 1,000 personyears, respectively). There were no significant differences between participants included and excluded from the analysis regarding gender (degrees of freedom $[\mathrm{df}]=1 ; \chi^{2}$ $=0.09 ; \mathrm{p}=0.752$ ) and education ( $\mathrm{df}=1 ; \chi^{2}=0.89 ; \mathrm{p}=$ $0.343)$, although the included participants were younger $\left(\mathrm{df}=1 ; \chi^{2}=4.33 ; p=0.03\right)$. Table 1 summarizes the baseline sociodemographic and other characteristics of the 1,281 participants with complete baseline information for all variables, divided according to cognitive impairment level.

\section{Association between cognitive impairment and all-cause mortality}

Table 2 shows the HR for all-cause mortality according to cognitive impairment for all participants and stratified according to gender. In the unadjusted HR model, the mortality risk was increased in participants with mild (HR: $1.71 ; 95 \%$ confidence interval $[95 \% \mathrm{Cl}] 1.36-2.15)$ and moderate cognitive impairment (HR: 2.50; 95\% Cl: 1.544.05). However, the associations did not remain significant in the final model ( $\mathrm{HR} \mathrm{MCl:} 1.15 ; 95 \% \mathrm{Cl} 0.89-1.48$ and HR moderate cognitive impairment: $1.35 ; 95 \% \mathrm{Cl}$ 0.81-2.24).

After gender stratification, only $\mathrm{MCl}$ increased the mortality risk in the unadjusted analysis (HR: 1.92; 95\% Cl 1.40-2.62), although it did not remain significant in the final model (HR: 1.35; 95\%Cl 0.96-1.88) for female participants. In male participants, mortality risk was increased by both $\mathrm{MCl}(\mathrm{HR} ; 1.42 ; 95 \% \mathrm{Cl} 1.01-2.00)$ and moderate cognitive impairment (HR: 3.30; 95\%Cl 1.696.45 ) in the unadjusted analysis, although only moderate cognitive impairment remained significant in the final model (HR: 2.30; 95\% Cl 1.12-2.4.74).

Figure 1 shows the estimated survivor function for the six curves (each cognitive functioning group for each sex). Compared to women with no cognitive impairment (the reference group), men with moderate cognitive impairment had the highest mortality risk (HR: 3.37; $95 \% \mathrm{Cl} 1.71-6.62)$ followed by men with $\mathrm{MCl}$ (HR: 1.55; $95 \% \mathrm{Cl} 1.05-2.25)$.

\section{Discussion}

Our results indicated that mild and moderate cognitive impairment, as determined by a single screening with the MMSE, were not associated with mortality risk in the entire sample after controlling for covariables. However, we found that gender did moderate mortality risk in this population, since men with moderate cognitive impairment had a significantly higher independent risk of mortality.

To our knowledge, only two other investigations, both in developed countries, have examined the association of cognitive decline and excess mortality using cognitive assessment similar to that of the present study. In the first, Park et al. ${ }^{8}$ investigated whether cognitive impairment is independently associated with increased longterm mortality in community-dwelling Koreans aged 60 years or older. At baseline, their participants were screened for cognitive impairment with the MMSE and were categorized into groups with no, mild, or moderate cognitive impairment. Their regression analysis included several variables, representing either confounders or mediators in the relationship between cognitive impairment (including different domain measurements) and mortality. Their results indicated that both mild and moderate cognitive impairment are associated with an increased risk of mortality. Although their sample was larger than ours $(2,712$ vs. 1,276$)$, the adjusted analysis was not rigorous enough to rule out confounders, since it did not include age, gender, education, exercise, current smoking, depression, osteoporosis, hyperlipidemia, and cerebrovascular diseases as factors. However, we used two approaches to investigate the extent to which a relationship between cognitive impairment and mortality was independent of potential confounding variables. First, we included numerous additional confounding factors, including APOE \&4 allele, impaired physical functioning, BMI, alcohol and caffeine consumption, and self-reported ethnicity. Second, we carried out a proportional hazards test, which was consistent with a constant HR over the follow-up period. 


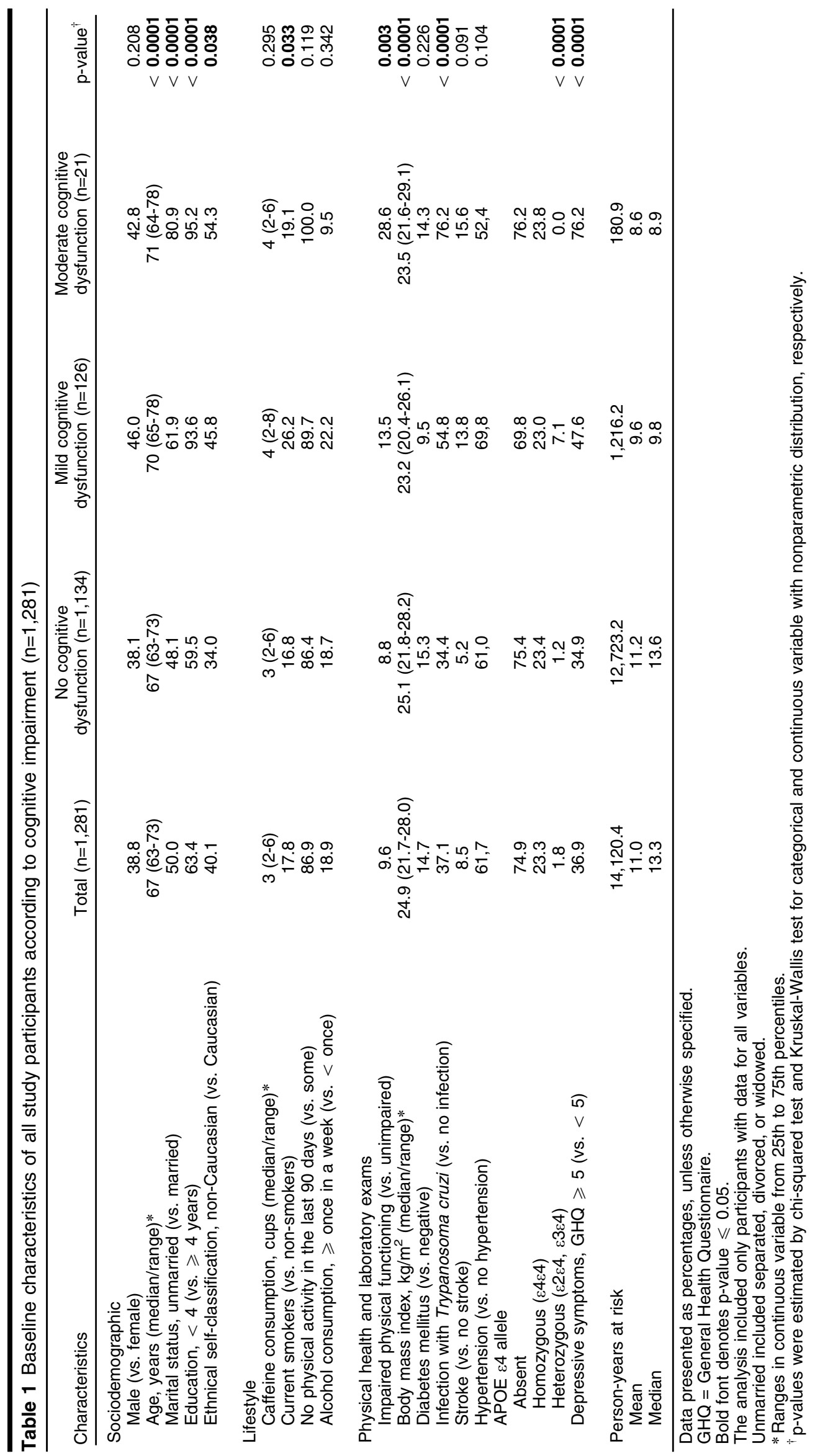




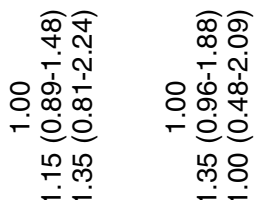

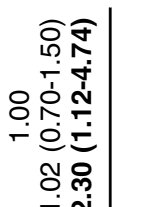

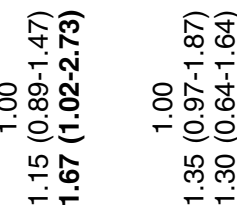

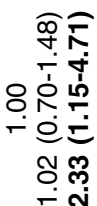

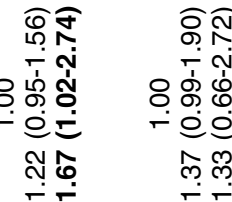
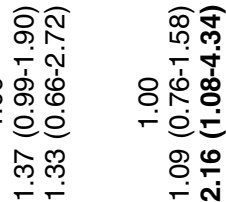

또똥

ง่่

ठ ले प़े

5
$\therefore$

중유

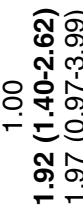

ง่

৪就

ชุ่อุ

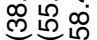

保

ช่

อลิ สุ

ษั่

ले के

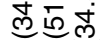

ल म -

लं 80

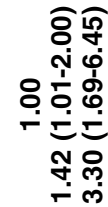

The second study, conducted by Bassuk et al. in New Haven, CT, USA, ${ }^{6}$ investigated the effects of cognitive impairment on excess mortality among community-dwelling adults aged 68 years or older, using MMSE scores to assess cognition. Their results indicated a strong relationship between both mild and severe cognitive impairment and mortality in participants under 80 years of age over a 3-year follow-up period. However, this study differed from ours in that MMSE scores were not assessed according to normative cognitive modeling, ${ }^{28}$ which reduces ceiling and floor effects and would be desirable in a study of the increasing burden of multiple forms of pathological damage to the brain. ${ }^{29}$ Thus, their results are not strictly comparable with ours.

Other investigations have assessed the association between cognition and mortality in different ways. A study by Paddick et al. in Tanzania, ${ }^{13}$ a low-income country, demonstrated that cognitive decline (i.e., $\mathrm{MCl}$ ) was associated with higher mortality rates than no cognitive impairment. In Indianapolis, IN, USA, Sachs et al. ${ }^{7}$ investigated whether cognitive impairment is independently associated with increased long-term mortality in primary care patients aged 60 years or older. At baseline, they screened patients for cognitive impairment with the Short Portable Mental Status Questionnaire, categorizing them into groups with no, mild, or moderate to severe cognitive impairment. They found that both mild and moderate to severe cognitive impairment are associated with an increased risk of mortality.

Our results showed an association between cognitive decline and excess mortality, particularly among men. Comparison with other studies could be compromised by methodological differences, such as study design, assessment instruments, sociocultural characteristics, healthcare access, treatment options, etc. However, such discrepancies were found even in the most directly comparable studies, in which cognitive impairment was measured and classified with the MMSE. ${ }^{6,8}$ Despite the methodological differences, however, our findings on gender differences agree with one German and two American studies in that men with cognitive impairment had shorter survival times. ${ }^{11,30,31}$

The mechanism for gender differences in the association between cognitive impairment and mortality risk has seldom been investigated. Some researchers have proposed that dementia severity is a less significant predictor of survival in women than in men. ${ }^{30}$

Alternatively, the same group proposed that the MMSE may be a more valid indicator of dementia severity in men than women, perhaps because social or cultural factors influence MMSE scores differently by sex. ${ }^{30}$ Furthermore, other mechanisms for dementia in men and women have been suggested, such as different profiles of comorbid illness and different causes of cognitive impairment. ${ }^{31}$

The association between high mortality risk and moderate cognitive impairment found in men could also be related to lifestyle differences with women. Univariate analysis revealed robust gender differences in alcohol consumption (40.1in men vs. $5.5 \%$ in women) and smoking (29.3 in men vs. $10.6 \%$ in women). Although several large epidemiological studies have reported a lower risk of 

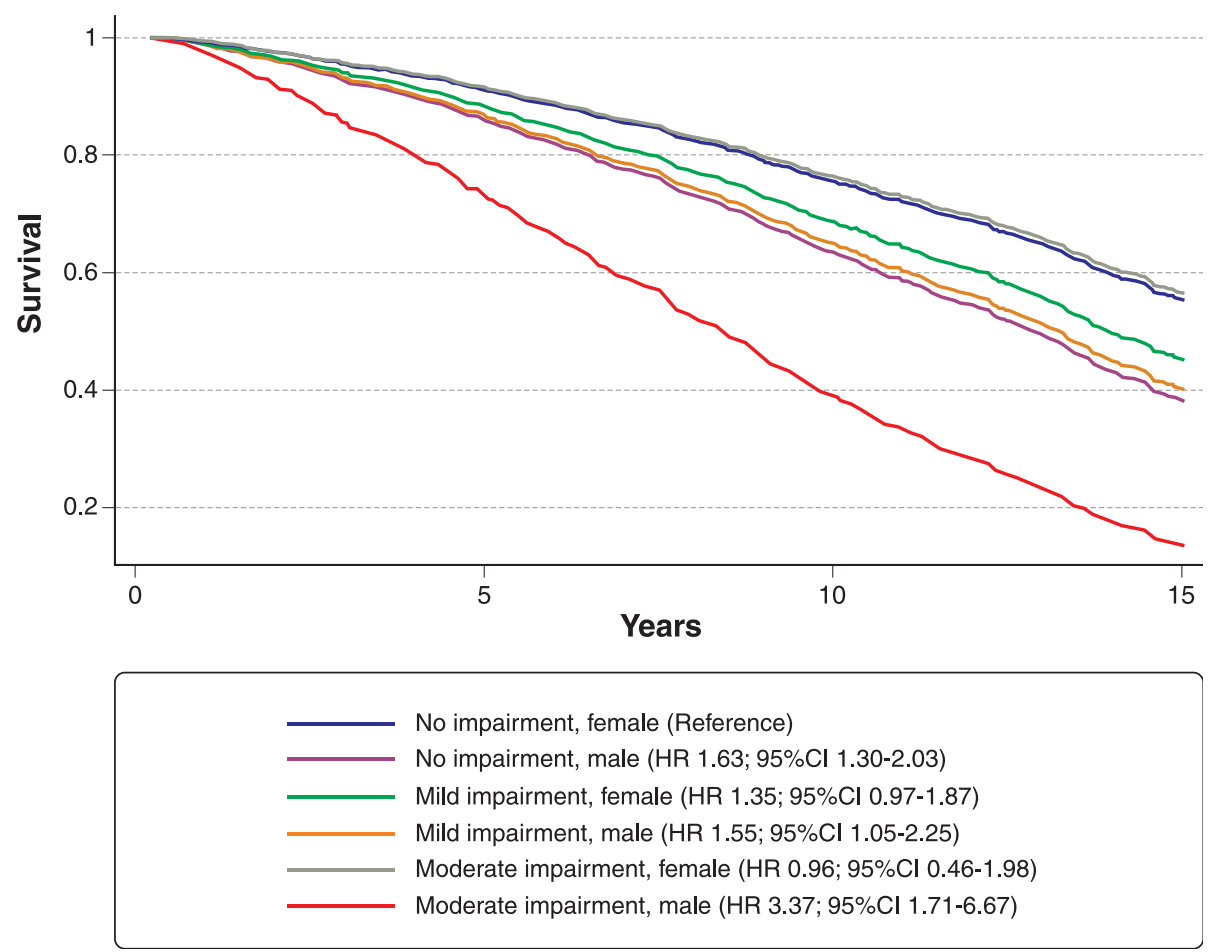

Figure 1 Cox's proportional hazards regression according to cognitive impairment group. $95 \% \mathrm{Cl}=95 \%$ confidence interval; $\mathrm{HR}=$ hazard ratios.

dementia in light to moderate drinkers than abstainers, ${ }^{32-35}$ a large longitudinal study in Norway recently reported a higher rate of dementia-related deaths in moderate drinkers than abstainers. ${ }^{36}$ This inconsistency has been ascribed to residual confounding, and Mendelian randomization has been recommended. ${ }^{37}$ The two studies that applied this technique found that moderate drinking has neither a protective effect on cognitive decline in older men (defined as MMSE $<23 / 30$; 6-year follow-up) ${ }^{38}$ nor a protective association with MMSE performance or word recall (cross-sectional study). ${ }^{37}$ Regarding smoking a risk factor for dementia and cognitive decline, a meta-analysis of 19 prospective studies ${ }^{39}$ found that current older smokers at baseline had an increased risk of dementia and cognitive decline than older adults who never or formerly smoked.

Our study is the first to address the association between cognitive impairment and mortality in South America, adding to the limited evidence from low- and middleincome countries. Its strengths include: 1) cognitive modeling based on MMSE scores, which reduces the influence of short-term changes in mood, pain, sleep, stress or physiological measures (such as serum glucose, blood pressure, and oxygen saturation); 2) the use of an MMSE version that has been validated for the Brazilian population; 3) mortality was determined over 15 years of follow-up in a large population-based cohort with a high response rate and minimal attrition; and 4) the analysis included adjustment for a range of potential confounders, including APOE $\varepsilon 4$ allele, impaired physical functioning, BMl, etc. The fact that the proportional hazards were consistent throughout the follow-up period further reduces the likelihood of confounding.

However, our study has some limitations that could affect interpretation of the results. Generalizability may be restricted because participants were obtained from a single city in southeastern Brazil, and most participants had low educational and socioeconomic status. However, the demographic characteristics of our study participants are very similar to the overall Brazilian population. Since cognition was only assessed at baseline, we could not evaluate the trajectory of cognitive impairment and its effects on mortality risk over the follow-up period, which was a significant weakness. The low accuracy of causeof-death coding in the mortality information system did not allow a deeper exploration of the data on this issue. There were only nine males in the moderate cognitive impairment group, which could have led to lower precision and power. Thus, the HR $(\mathrm{Cl})$ was not as high as expected. Finally, the HR (Cl) for moderate cognitive impairment in men and women overlapped. Statisticians have traditionally considered $\mathrm{Cl}$ overlap between two independent groups to demonstrate no statistically significant difference between them. ${ }^{40-42}$ However, this discussion has referred to normal populations and symmetrical $\mathrm{Cl}$ for sample means. When comparing proportions bounded by categories (as we did), such Cls are generally asymmetrical, i.e., that the lower and upper arms are of unequal length. ${ }^{33}$ In such cases, overlapping $\mathrm{Cl}$ could indicate a statistically significant difference when it is about half the average of arm length or $p \leqslant 0.01$, as we found in our analysis. ${ }^{41}$ 
In conclusion, our findings suggest an association between moderate cognitive impairment and all-cause mortality in men in a large Brazilian cohort of older adults. However, future studies are needed to both replicate these results, determine which mechanisms underly this phenomenon, and determine whether there are effective measures to reduce mortality in such cases.

\section{Acknowledgements}

The Bambui Cohort Study of Aging was carried out by a team of researchers based at the Fundação Oswaldo Cruz in the state of Minas Gerais, Brazil. Funding was provided by the Financiadora de Estudos e Projetos (FINEP), the Conselho Nacional de Desenvolvimento Científico e Tecnológicos (CNPq), and the Fundação de Amparo à Pesquisa do Estado de Minas Gerais (FAPEMIG). JOAF, MFL-C, BSD, and SLB received grants from CNPq (305274/2014-7). BSD currently receives research support from the National Institute of Mental Health (MH114970-01, MH115953-01, and R03MH115212-01).

\section{Disclosure}

The authors report no conflicts of interest.

\section{References}

1 Richardson C, Stephan BC, Robinson L, Brayne C, Matthews FE; Cognitive Function and Ageing Study Collaboration. Two-decade change in prevalence of cognitive impairment in the UK. Eur J Epidemiol. 2019;34:1085-92.

2 Winblad B, Palmer K, Kivipelto M, Jelic V, Fratiglioni L, Washlund LO, et al. Mild Cognitive Impairment - beyond controversies, towards a consensus: report of the International Working Group on Mild Cognitive Impairment. J Intern Med. 2004;256:240-46.

3 Petersen RC, Roberts RO, Knopman DS, Boeve BF, Gefa YE Ivnik $\mathrm{Rj}$, et al. Mild cognitive impairment: ten years later. Arch Neurol. 2009;66:1447-55.

4 American Psychiatric Association. Diagnostic and Statistical Manual of Mental Disorders, Fifth Edition (DSM-5).Arlington: American Psychiatric Publishing; 2013.

5 Albert MS, DeKosky ST, Dickson D, Dubois B, Feldman HH, Fox NC et al. The diagnosis of mild cognitive impairment due to Alzheimer's disease: recommendations from the National Institute on Aging-Alzheimer's Association workgroups on diagnostic guidelines for Alzheimer's disease. Alzheimers Dement. 2011;7:270-9.

6 Bassuk SS, Wypij D, Berkman LF. Cognitive impairment and mortality in the community-dwelling elderly. Am J Epidemiol. 2000;151:676-88.

7 Sachs GA, Carter R, Holtz LR, Smith F, Stump TE, Tu W, et al. Cognitive impairment: an independent predictor of excess mortality: a cohort study. Ann Intern Med. 2011;155:300-8.

8 Park MH, Kwon DY, Jung JM, Han C, Jo I, Jo SA. Mini-mental status examination as predictors of mortality in the elderly. Acta Psychiatr Scand. 2013;127:298-304.

9 Santabárbara J, Gracia-García P, Pírez G, López-Antón R, De La Cámara $\mathrm{C}$, Ventura $\mathrm{T}$, et al. Mortality in mild cognitive impairment diagnosed with DSM- 5 criteria and with Petersen's criteria: A 17-year followup in a community study. Am J Geriatr Psychiatry. 2016;24:977-86.

10 de Bruijn RF, Akoudad S, Cremers LG, Hofman A, Niessen WJ, van $\operatorname{der}$ Lugt A, et al. Determinants, MRI correlates, and prognosis of mild cognitive impairment: the Rotterdam study. J Alzheimers Dis. 2014;42 Suppl 3: S239-49.

11 Perna L, Wahl HW, Mons U, Saum KU, Holleczek B, Brenner H. Cognitive impairment, all-cause and cause-specific mortality among non-demented older adults. Age Ageing. 2015;44:445-51.
12 Vassilaki M, Cha RH, Aakre JA, Therneau TM, Geda YE, Mielke MM, et al. Mortality in mild cognitive impairment varies by subtype, sex, and lifestyle factors: the Mayo Clinic Study of Aging. J Alzheimers Dis. 2015;45:1237-45.

13 Paddick SM, Kisoli A, Dotchin CL, Gray WK, Chaote P, Longdon A, et al. Mortality rates in community-dwelling Tanzanians with dementia and mild cognitive impairment: a 4-year follow-up study. Age Ageing. 2015;44:636-41.

14 Stephan BC, Matthews FE, McKeith IG, Bond J, Brayne C; Medical Research Council Cognitive Function and Aging Study. Early cognitive change in the general population: how do different definitions work? J Am Geriatr Soc. 2007;55:1534-40.

15 Hogan DB, Ebly EM. Primitive reflexes and dementia: results from the Canadian study of health and aging. Age Ageing. 1995;24:375-81.

16 Bruscoli M, Lovestone S. Is MCl really just early dementia? A systematic review of conversion studies. Int Psychogeriatr. 2004;16:129-40.

17 Mitchell AJ, Shiri-Feshki M. Rate of progression of mild cognitive impairment to dementia -- meta-analysis of 41 robust inception cohort studies. Acta Psychiatr Scand. 2009;119:252-65.

18 Matthews FE, Stephan BC, McKeith IG, Bond J, Brayne C; Medical Research Council Cognitive Function and Aging Study. Two-year progression from mild cognitive impairment to dementia: to what extent do different definitions agree? J Am Geriatr Soc. 2008;56: 1424-33.

19 Lima-Costa MF, Firmo JO, Uchoa E. Cohort profile: the Bambuí (Brazil) cohort study of ageing. Int J Epidemiol, 2011;40:862-7.

20 Seabra MLV, Concilio GV, Villares JB, Carlini EA. Avaliação do teste "Mini-mental state" em voluntários e pacientes brasileiros. Rev ABPAPL. 1990;12:1-7.

21 Folstein MF, Folstein SE, McHugh PR. Mini-mental state. A practical method for grading the cognitive state of patients for the clinician. $J$ Psychiatr Res. 1975;12:189-98.

22 Castro-Costa E, Fuzikawa C, Ferri C, Uchoa E, Firmo J, Lima-Costa $M F$, et al. Dimensions underlying the mini-mental state examination in a sample with low-education levels: the Bambui health and aging study. Am J Geriatr Psychiatry. 2009;17:863-72.

23 Castro-Costa E, Dewey ME, Uchôa E, Firmo JO, Lima-Costa M F, Stewart R. Construct validity of mini-mental state examination across time in a sample with low-education levels: 10-year follow-up of the Bambuí cohort study of ageing. Int J Geriatr Psychiatry. 2014;29: 1294-303.

24 American Diabetes Association. Classification and diagnosis of diabetes: Standards of Medical Care in Diabetes. Diabetes Care. 2018; 41(Suppl. 1): S13-27.

25 Plan and operation of the Third National Health and Nutrition Examination Survey, 1988-94. Series 1: programs and collection procedures. Vital Health Stat 1. 1994;(32): 1-407.

26 Chobanian AV, Bakris GL, Black HR, Cushman WC, Green LA, Izzo $\mathrm{JL} \mathrm{Jr}$, et al. The seventh report of the joint national committee on prevention, detection, evaluation, and treatment of high blood pressure: the JNC 7 report. JAMA. 2003;289:2560-72.

27 Costa E, Barreto SM, Uchoa E, Firmo JO, Lima-Costa MF, Prince M. Is the GDS-30 better than the GHQ-12 for screening depression in the elderly people in the community? The Bambui health aging study (BHAS). Int Psychogeriatr. 2006;18:493-503.

28 Steinerman JR, Hall CB, Sliwinski MJ, Lipton RB. Modeling cognitive trajectories within longitudinal studies: a focus on older adults. J Am Geriatr Soc. 2010;58 Suppl 2: S313-8.

29 Jack CR Jr, Knopman DS, Jagust WJ, Shaw LM, Aisen PS, Weiner $\mathrm{MW}$, et al. Hypothetical model of dynamic biomarkers of the Alzheimer's pathological cascade. Lancet Neurol. 2010;9:119-28.

30 Moritz DJ, Fox PJ, Luscombe FA, Kraemer HC. Neurological and psychiatric predictors of mortality in patients with Alzheimer disease in California. Arch Neurol. 1997;54:878-85.

31 Perls TT, Morris JN, Ooi WL, Lipsitz LA. The relationship between age, gender and cognitive performance in the very old: the effect of selective survival. J Am Geriatr Soc. 1993;41:1193-201.

32 Topiwala A, Ebmeier KP. Effects of drinking on late-life brain and cognition. Evid Based Ment Health. 2018;21:12-5.

33 Ruitenberg A, van Swieten JC, Witteman JC, Mehta KM, van Duijn $\mathrm{CM}$, Hofman A, et al. Alcohol consumption and risk of dementia: the Rotterdam Study. Lancet. 2002;359:281-6. 
34 Mukamal KJ, Kuller LH, Fitzpatrick AL, Longstreth WT Jr, Mittleman MA, Siscovick DS. Prospective study of alcohol consumption and risk of dementia in older adults. JAMA. 2003;289:1405-13.

35 Heffernan M, Mather KA, Xu J, Assareh AA, Kochan NA, Reppermund $S$, et al. Alcohol consumption and incident dementia: evidence from the Sydney memory and ageing study. J Alzheimers Dis. 2016;52:529-38.

36 Ormstad H, Rosness TA, Bergem AL, Bjertness E, Strand BH; GENIDEM-Group. Alcohol consumption in the elderly and risk dementia-related death--a Norwegian prospective study with a 17year follow-up. Int J Neurosci. 2016;126:135-44.

37 Au Yeung SI, Jiang C, Cheng KK, Cowling BJ, Liu B, Zhang W, et al. Moderate alcohol use and cardiovascular disease from Mendelian randomization. PLoS One. 2013;8:e68054.
38 Almeida OP, Hankey GJ, Yeap BB, Golledge J, Flicker L. Alcohol consumption and cognitive impairment in older men: a mendelian randomization study. Neurology. 2014;82:1038-44.

39 Anstey KJ, von Sanden C, Salim A, O'Kearney R. Smoking as a risk factor for dementia and cognitive decline: a meta-analysis of prospective studies. Am J Epidemiol. 2007;166:367-78.

40 Cumminng G, Finnch S. Inference by eye: confidence intervals and how to read pictures of data. Am Psychol. 2005;60:170-80.

41 Cumming G. Inference by eye: Reading the overlap of independent confidence intervals. Stat Med. 2009;28:205-20.

42 Nakagawa S, Cuthill IC. Effect size, confidence interval and statistical significance: a practical guide for biologists. Biol Rev Camb Philos Soc. 2007;82:591-60. 